\title{
Cosmic ray energy changes at the termination shock and in the heliosheath
}

\author{
H. Moraal*, R.A. Caballero-Lopez ${ }^{\dagger}$, K.G. McCracken**, F.B. McDonald**, \\ R.A. Mewaldt ${ }^{\ddagger}$, V. Ptuskin ${ }^{\S}$ and M.E. Wiedenbeck ${ }^{\mathbb{I I}}$ \\ ${ }^{*}$ School of Physics, North-West University, Potchefstroom 2520, South Africa \\ ${ }^{\dagger}$ Instituto de Geofísica, Universidad Nacional Autónoma de México, 04510, México \\ ${ }^{* *}$ Institute for Physical Science and Technology, Univ. of Maryland, College Park, MD, 20742, USA \\ ${ }^{*}$ Space Radiation Laboratory, California Institute of Technology, Pasadena, CA 91125, USA \\ $\S$ IZMIRAN, Troitsk, Moscow Region 142190, Russia \\ ${ }^{I}$ Jet Propulsion Laboratory, California Institute of Technology, Pasadena, CA 91109, USA
}

\begin{abstract}
Voyager 1 crossed the termination shock of the solar wind in December 2004 at 94 $\mathrm{AU}$ and currently measures the cosmic ray intensity in the heliosheath. To better understand this modulation region beyond the shock, where adiabatic energy changes should be small, we review the net effect of energy changes during the modulation process, including adiabatic deceleration in the solar wind, acceleration at the termination shock, and the possibility that stochastic acceleration in the heliosheath may also make a contribution.
\end{abstract}

Keywords: Cosmic ray spectra, cosmic ray composition, termination shock, heliosheath

PACS: $96.50 . \mathrm{sb}, 96.50 . \mathrm{sh}, 96.50 . \mathrm{Ek}$

\section{INTRODUCTION}

Two recent observations justify a renewed assessment of energy changes in cosmic ray modulation, First, Voyager 1 made two interactions with the solar wind termination shock (SWTS) in 2002/03 and 2003/04, e.g. [6], [8], and [9], and crossed it on 16 December 2004 at 94 AU, [1], [4], [14]. Particle spectra at the shock and in the heliosheath region were not of the expected power law form with an exponential cutoff, but only gradually approached this form several months after the shock crossing. Stone et al. [14] stated that to understand these spectra, a fundamental change in the source model of ACRs may possibly be required. Several papers in this volume study this problem. Here we investigate whether the process of stochastic acceleration in the heliosheath may make a contribution to this.

A different, more general issue is that spectral shapes provide a tool to study cosmic ray properties, e.g. the difference between secondary and primary spectra which reflects propagation, nuclear fragmentation and escape of cosmic rays from the galaxy. A specific case is that of $\mathrm{K}$-capture secondaries such as ${ }^{51} \mathrm{~V}$, being produced from ${ }^{51} \mathrm{Cr}$, as described by [7] and [11]. Such processes are best studied at $T \leq 1 \mathrm{GeV} / \mathrm{n}$, where good statistical accuracy can be achieved, but this requires that modulation effects, including energy changes, must first be unfolded before the spectral shape in interstellar space can be determined. We show that acceleration by the SWTS can have a significant effect on the ratios of these K-capture electron secondaries observed in the inner heliosphere, and that this serves as a diagnostic tool to infer unmodulated spectra in the galaxy.

CP 858, Physics of the Inner Heliosheath,

edited by J. Heerikhuisen, V. Florinski, G. P. Zank, and N. V. Pogorelov

(C) 2006 American Institute of Physics 978-0-7354-0355-0/06/\$23.00 


\section{ENERGY CHANGE CALCULATIONS}

To study these observations we consider numerical solutions of the cosmic ray transport equation (TPE) for the evolution of the distribution function $f$ in terms of momentum $p$ :

$$
\frac{\partial f}{\partial t}+\mathbf{V} \cdot \nabla f-\nabla \cdot(\mathrm{K} \cdot \nabla f)-\frac{1}{3}(\nabla \cdot \mathbf{V}) \frac{\partial f}{\partial \ln p}-\frac{1}{p^{2}} \frac{\partial}{\partial p}\left(p^{2} D \frac{\partial f}{\partial p}\right)=Q .
$$

Here $\mathbf{V}$ is the solar wind velocity and $\mathrm{K}(\mathbf{r}, P, t)(P=$ rigidity $)$ the diffusion tensor with elements $\kappa_{\|}(\mathbf{r}, P, t)$ and $\kappa_{\perp}(\mathbf{r}, P, t)$ for scattering along and perpendicular to the HMF, $\mathbf{B}$, together with an antisymmetric coefficient $\kappa_{T}=\beta P /(3 B)$ for gradient, curvature, neutral sheet, and shock drift. The radial and latitudinal components are $\kappa_{r r}=\kappa_{\|} \cos ^{2} \psi+$ $\kappa_{\perp} \sin ^{2} \psi, \kappa_{\theta \theta}=\kappa_{\perp}$. The HMF is the standard Parker spiral with a strength at Earth varying from 5 to $10 \mathrm{nT}$ from solar minimum (SMIN) to maximum (SMAX). The SWTS, with compression ratio $s$, is put at $r_{s}=90 \mathrm{AU}$, with the outer boundary, $r_{b}$, corresponding to the heliopause at $150 \mathrm{AU}$. Inside the shock $V=400 \mathrm{kms}^{-1}$ while in the heliosheath $V=(400 / s)\left(r_{s} / r\right)^{2} \mathrm{kms}^{-1}$. It increases with a factor of 2 between latitudes of $20^{\circ}$ and $30^{\circ}$ during SMIN.

The last term on the left of (1) describes diffusion in momentum space. In the solar wind it is small compared to adiabatic losses and is usually neglected. In the last section it is pointed out, however, that it may be significant in the heliosheath.

Figure 1 shows mean energy changes calculated in various levels of sophistication of the model, with the detailed calculations described in [3]. In A and B there is no shock, meaning that the supersonic wind extends out to $r_{b}=150 \mathrm{AU}$. Thus, the energy losses of these panels are standard, well-known results. In the Force-Field (FF) and one-dimensional spherically symmetric (1D/SS) solution $\kappa_{r r}=6 \times 10^{22} \beta P(\mathrm{GV})(=$ $2.4 \times 10^{22} \beta$ if $\left.P<0.4 \mathrm{GV}\right) \mathrm{cm}^{2} \mathrm{~s}^{-1}$, producing a FF parameter [5] $\phi=\int V d r / 3 \kappa_{1}=$ $500 \mathrm{MV}$, and a consequent mean energy loss $\left\langle T^{*}\right\rangle-T=500 \mathrm{MeV}$ for protons, independent of energy $\left(\kappa_{1}\right.$ is the spatial part of $\left.\kappa_{r r}\right)$. Other energy changes are compared with this widely used baseline value. The 1- $\sigma$ band shown for the 1D/SS solution in A signifies that there is a wide energy dispersion due to the diffusive transport mode. B shows the results for a 2D (radial distance, polar angle) solution that allows latitudinal diffusion through $\kappa_{\theta \theta}=\kappa_{\perp}=10 \%$ of $\kappa_{r r}$. The energy loss in the two drift cases is significantly less than in the no-drift case because drift allows easier access to Earth. Generally, however, the various no-shock solutions of A and B show energy losses within a factor of 2 of one another. On the other hand, in C and D a SWTS is inserted at $r_{s}=90 \mathrm{AU}$, for a moderate $(s=3)$ and strong $(s=4)$ shock respectively. At the shock $\kappa_{r r}, \kappa_{\theta \theta}$ and $V$ all drop by a factor of $s$, while $V$ decreases further $\propto r^{-2}$ in the heliosheath. The energy loss is now dramatically less, especially at higher energies. Since the parameters inside the shock are identical to those in B, all differences between $\mathrm{C}$ and $\mathrm{D}$ relative to $\mathrm{B}$ are due to the acceleration on the shock and the absence of adiabatic losses beyond $r_{s}$. Thus, in a heliosphere with a SWTS and heliosheath a given amount of modulation is accompanied by much less energy loss, particularly at high energies ( $\geq 1$ $\mathrm{GeV}$ ) where the acceleration at the SWTS becomes larger than the loss in the upstream supersonic solar wind, producing a net gain. 


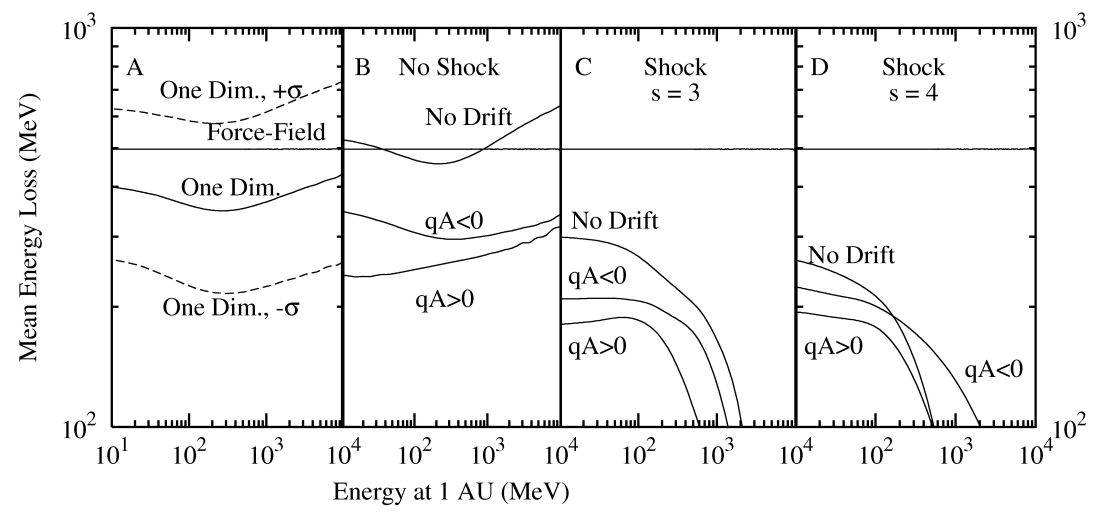

FIGURE 1. Mean energy losses, $\Delta T=<T^{*}>-T$, suffered by particles observed at Earth. (A) ForceField energy loss compared to that of a one-dimensional (spherically symmetric) solution of the TPE with the same parameters (see text). The dashed lines indicate the $\pm 1 \sigma$ band from within which $68 \%$ of the particles that have energy $T$ at Earth, originate in the LIS. (B) Two-dimensional solution with no shock, for the no-drift and qA $>0$ and qA $<0$ drift cases. (C) and (D) Same as (B) but for the shock-heliosheath solution with a moderate $(\mathrm{s}=3)$ and strong $(\mathrm{s}=4)$ shock inserted at $r_{s}=90 \mathrm{AU}$.

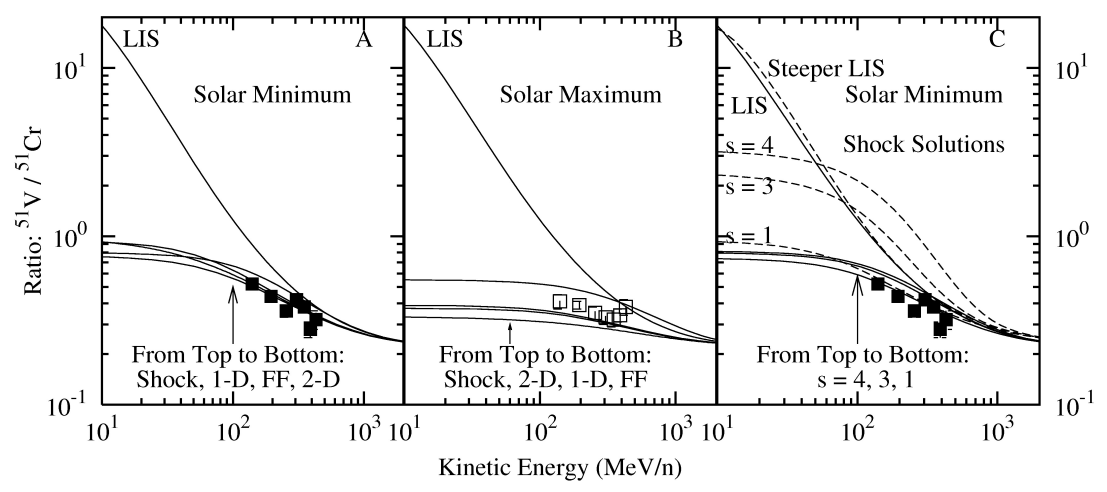

FIGURE 2. The ratio ${ }^{51} \mathrm{~V} /{ }^{51} \mathrm{Cr}$ at Earth for SMIN (A) and SMAX (B). Observations are from ACE by [11], for 97/08/28-99/08/17 and 00/02/24-03/01/05. Calculated ratios are with $V, r_{b}$ and $r_{s}$ the same as in Figure 1. In (A) the no-shock FF and 1D/SS solutions have $\kappa_{r r}=1.2 \times 10^{23} \beta P\left(\mathrm{GV} \mathrm{cm}^{2} \mathrm{~s}^{-1}\right.$, producing $\phi=250 \mathrm{MV}$; the no-shock 2D solution and the shock solution (for a moderate shock with $s=3$ ) are for qA>0 drift with $\kappa_{\theta \theta}=80 \%$ of $\kappa_{r r}$, and with $\kappa_{r r}$ decreasing with a factor 2 toward the poles. In (B) the $\kappa \mathrm{s}$ are reduced such that $\phi=1135 \mathrm{MV}$. The full lines in (C) are for the same parameters as the shock solution of (A) but for three values of $s$. The dashed lines are with the ${ }^{51} \mathrm{Cr}$ and ${ }^{51} \mathrm{~V}$ LIS both multiplied by $\left(0.22+61 / T+7000 / T^{2}\right) \exp (-14.9 / T)(T$ in $\mathrm{MeV} / \mathrm{n})$, keeping their ratio (almost) constant.

\section{K-CAPTURE ELECTRON SECONDARIES}

Niebur et al. [11] and Mewaldt et al. [7] studied the ${ }^{51} \mathrm{~V} /{ }^{51} \mathrm{Cr}$ ratio, which is strongly energy dependent because at low energies the production of ${ }^{51} \mathrm{~V}$ by K-capture of electrons 
in ${ }^{51} \mathrm{Cr}$ ions is much faster than at high energies. This energy dependence is modified by cosmic ray modulation. The authors observed that the ratio is less energy dependent at SMAX than at SMIN, and interpreted this as due to different amounts of energy loss suffered at these periods, namely $<\Delta T>\approx 400 \mathrm{MeV} / \mathrm{n}$ and $\approx 200 \mathrm{MeV} / \mathrm{n}$ respectively. They only used a 1D/SS solution of the TPE, however, and in view of the much different amounts of energy change in a more realistic heliosphere as shown above, we repeat this calculation with more extensive solutions.

Figures $2 \mathrm{~A}$ and $2 \mathrm{~B}$ show ${ }^{51} \mathrm{~V} /{ }^{51} \mathrm{Cr}$ ratios using an LIS ratio (solid lines) that fits the composition of $4 \leq \mathrm{Z} \leq 28$ species, see e.g. [15] and [7]. Four modulation solutions are also shown in each panel, the long dashed one being the full shock-plus-heliosheath solution. The other three are for progressively simpler models to demonstrate the significance of the various effects. The low-energy modulated ratios are much lower than the LIS ratio. This is due to adiabatic energy loss, because this produces copious amounts of low-energy ${ }^{51} \mathrm{Cr}$ at $1 \mathrm{AU}$, far exceeding that in the LIS. Note that at SMAX the 1 AU ratio at $T>400 \mathrm{MeV} / \mathrm{n}$ is higher than the LIS value. This is a shock acceleration effect: low-energy ${ }^{51} \mathrm{Cr}$ and ${ }^{51} \mathrm{~V}$ particles are accelerated up to these energies, but because there are fewer low-energy ${ }^{51} \mathrm{Cr}$ than ${ }^{51} \mathrm{~V}$ particles to be accelerated, the ratio at high energies $(T>400 \mathrm{MeV} / \mathrm{n})$ increases. The dash-dot solution is for the same model, but without a shock and with the supersonic solar wind continuing unabated up to $r_{b}=$ $150 \mathrm{AU}$. This change has a small effect at SMIN, but it decreases the SMAX ratio by $\approx 30 \%$. The ratio is now less energy dependent, due to the additional adiabatic energy loss suffered in the region between 90 and $150 \mathrm{AU}$ that 'stretches out' the ratio more evenly towards low energies. The dotted solution is the 1D/SS solution of [2], [7] and [11]. The differences between the 1D/SS and other solutions at SMIN are small, and the data points are not sufficiently accurate to discriminate among them. Thus, the most dominant modulation mechanisms are radially inward diffusion and outward convection, as captured by the FF and 1D/SS solutions. Latitudinal diffusion and drift only play a secondary, modifying role. The only solution that differs significantly from the others is the shock-plus-heliosheath one at SMAX in Figure 2B. Note that for $s=3$ it lies above, while the no-shock solutions all lie below the data points. This suggests that at least a weak shock is more consistent with the data than no shock.

The above analysis, therefore, shows that the ${ }^{51} \mathrm{~V} /{ }^{51} \mathrm{Cr}$ ratio is heavily modulated from its interstellar value, but that it is not overly sensitive to the detailed properties of the heliosphere that determine the cosmic transport parameters. This is, however, only true for realistic LISs that have low intensities at low energies. There is a strong sensitivity to the form of the LIS in the range $T<10 \mathrm{MeV} / \mathrm{n}$, as can be seen from Figure 2C where both the ${ }^{51} \mathrm{Cr}$ and ${ }^{51} \mathrm{~V}$ LIS are multiplied by $\left(0.22+61 / T+7000 / T^{2}\right) \exp (-14.9 / T)(T$ in $\mathrm{MeV} / \mathrm{n})$. At $T \geq 200 \mathrm{MeV} / \mathrm{n}$ this has little effect, but at low energies this lets the LISs increase with decreasing energy, such that at $10 \mathrm{MeV} / \mathrm{n}$ the intensity is 4000 times that of the spectra used in Figure $2 \mathrm{~B}$. The results for the modulated ${ }^{51} \mathrm{~V} /{ }^{51} \mathrm{Cr}$ ratio for these steeper LISs are shown by the dashed lines in Figure 2C, while the full-line solutions are for the same LISs as in Figure 2A. This time only solutions for the full shock-plusheliosheath model are shown, for three values of the shock compression ratio, $s=4,3$, and 1 . The overall message is that (a) the modulated ${ }^{51} \mathrm{~V} /{ }^{51} \mathrm{Cr}$ ratio depends strongly on the form of the low-energy LIS of these species and not just on the LIS ratio, and (b) if these low-energy spectra are steep, the ratio also depends sensitively on the strength of 
the shock. These two effects can be understood as follows. If the spectrum at $T<100$ $\mathrm{MeV} / \mathrm{n}$ is steep, it means that many low-energy particles are available for acceleration to $T>100 \mathrm{MeV} / \mathrm{n}$, and this acceleration is so efficient that it overtakes adiabatic cooling, lifting the ratio above the LIS value. Also, this effect stops at $T \approx 1 \mathrm{GeV} / \mathrm{n}$ due to the natural cutoff of the acceleration at these energies for a shock of these dimensions, as was discussed by [13]. Conversely, when the low-energy spectra are flat, or diminish with decreasing energy, there are very few particles available for acceleration, and the full lines in Figure 2C show that, although the acceleration has the same efficiency, it has little effect on the spectra at $1 \mathrm{AU}$. The observations clearly favor low-energy spectra that turn down with decreasing energy.

\section{STOCHASTIC ACCELERATION IN THE HELIOSHEATH}

As mentioned in the Introduction, we also investigate whether stochastic (or 2nd order Fermi) acceleration in the heliosheath, described by the last term on the left of (1), may contribute to the puzzling spectral shapes observed by, e.g., [4] and [14] on Voyager 1 after it crossed the SWTS. The solid symbols in Figure 3 demonstrate this point for He, namely that these spectra do not unfold into the expected power law with an exponential cutoff. The data points above $100 \mathrm{MeV} / \mathrm{n}$ are GCRs, which is not controversial and will not receive further attention here. Those between 8 and $100 \mathrm{MeV} / \mathrm{n}$ are thought to be ACRs, but the puzzle is why they still seem to be modulated on the shock. Those below $8 \mathrm{MeV} / \mathrm{n}$ are so-called termination shock particles (TSPs), believed to be accelerated locally on the shock. Among the various explanations for the combined TSP plus ACR component, we show here that if the TSPs are accelerated on the SWTS and convected into the heliosheath, they can serve as source for stochastic acceleration there, to qualitatively produce the observed spectral shape.

Inside the SWTS this stochastic acceleration is unimportant. The Alfvén speed is $V_{A}=B /\left(\mu_{0} \rho\right)^{1 / 2}$, with $\rho$ the solar wind density. At 1 AU the value is $V_{A} \approx 40 \mathrm{~km} / \mathrm{s}$. For $r>>1 \mathrm{AU}, B \propto r^{-1}$, and since $\rho \propto r^{-2}, V_{A}$ is independent of $r$. According to [12] the diffusion coefficient for stochastic acceleration is $D=p^{2} V_{A}^{2} /\left(9 \kappa_{||}\right)$. For a typical $\kappa_{||}=6 \times 10^{22} \beta P(\mathrm{GV})\left(=2.4 \times 10^{22} \beta\right.$ if $\left.P<0.4 \mathrm{GV}\right) \mathrm{cm}^{2} / \mathrm{s}$ at Earth, the time scale for this acceleration is $\tau_{\text {stoch }} \sim p^{2} / D=9 \kappa_{||} / V_{A}^{2} \approx 4 \times 10^{5} \beta P(\mathrm{GV})$ days. To be a viable process, this time must be comparable to other time scales in (1). The time for propagation out to the SWTS at $r_{s}=90 \mathrm{AU}$ is $\tau_{\mathrm{conv}} \sim r / V \approx 390$ days. That for acceleration by the SWTS to a power law is $\tau_{\text {shock }} \sim \kappa / V^{2} \approx 430 \beta P(\mathrm{GV})$ days. (Perpendicular diffusion is the dominant process here, and from our experience with solutions of (1) this should be of the same order of magnitude as $\kappa_{\|}$at $1 \mathrm{AU}$ as given above. Thus, for typical 80 $\mathrm{MeV}$ protons $(\beta P=0.15 \mathrm{GV}), \tau_{\text {shock }}=64$ days $<\tau_{\text {conv }}=390$ days $<\tau_{\text {stoch }}=60000$ days, so that the stochastic process is much too slow to be effective.

The situation is quite different in the heliosheath. If $V_{o}$ and $B_{o}$ are the wind and HMF values immediately inside the shock, their profile in the heliosheath is $V=\left(V_{o} / s\right)\left(r_{s} / r\right)^{2}$ for incompressible subsonic flow, and $B=B_{o} s\left(r / r_{s}\right)$ if the Parker field is maintained. Thus the downstream Alfvén speed is $V_{A}=40 \sqrt{s}\left(r / r_{s}\right) \mathrm{km} / \mathrm{s}$, and if $\kappa$ in the heliosheath

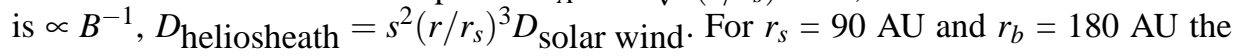




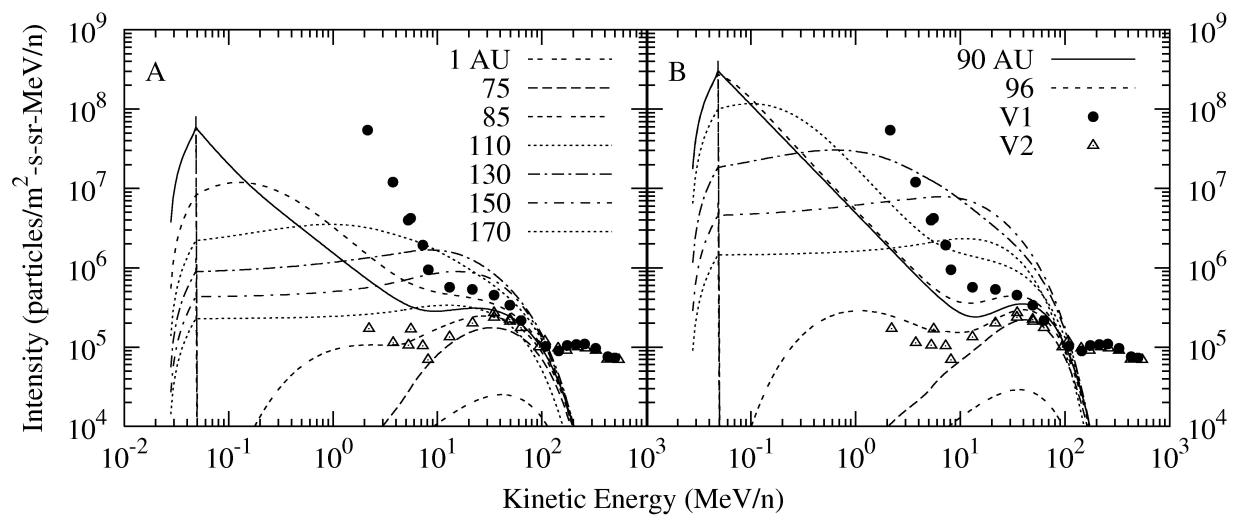

FIGURE 3. Voyager 1 and 2 spectra from 16 to 23 in January 2005, together with solutions of the TPE (1), containing stochastic acceleration. The full line is the shock spectrum at $90 \mathrm{AU}$. Below $10 \mathrm{MeV} / \mathrm{n}$ it mainly consists of TSPs, while at higher energies it mainly consists of particles that were stochastically accelerated in the outer heliosheath. In panels A and B the coefficient $D$ for stochastic acceleration is multiplied by $\left(r / r_{b}\right)^{2}$ and $\left(r / r_{b}\right)^{6}$ to concentrate this acceleration in the outer regions of the heliosheath.

convection time in the heliosheath is $\tau_{\mathrm{conv}}=\int d r / V \approx 3600$ days, almost 10 times longer than from the sun to the SWTS. On the other hand, it follows from the same calculation as above that for $80 \mathrm{MeV} / \mathrm{n}$ particles $\tau_{\text {stoch }} \approx 230$ days at $r_{b}=180 \mathrm{AU}$. This is so fast in comparison with $\tau_{\text {conv }}$ that stochastic acceleration in the outer regions of the heliosheath seems quite viable.

We therefore model the three-component V1 spectrum as follows: In this demonstration solution of the TPE (1) we do not pay attention to the GCR component, thus taking the LIS on the outer boundary at $r_{b}=180$ AU zero. We take a mono-energetic source $Q$ at $0.05 \mathrm{MeV} / \mathrm{n}$, a moderate $(s=2.5)$ shock, producing a power law, $j_{T} \propto T^{-1.5}$ up to $\approx 10$ $\mathrm{MeV} / \mathrm{n}$ (full line) at the SWTS at $90 \mathrm{AU}$. In the heliosheath these particles get strongly accelerated by the stochastic term in (1). In this simplest possible demonstration, we use a spherically symmetric heliosphere with $\kappa=6 \times 10^{22} \beta P(\mathrm{GV}) \mathrm{cm}^{2} / \mathrm{s}$, independent of $r$ for $r<r_{s}$, dropping with $s\left(r_{s} / r\right)$ for $r>r_{s}$. (Once again, $\kappa \propto \beta$ if $P<0.4 \mathrm{GV}$.) $D$ is calculated as above, but in panel A it is multiplied by $\left(r / r_{b}\right)^{2}$ and in B with $\left(r / r_{b}\right)^{6}$ to make the stochastic acceleration preferentially efficient in the outer heliosheath. The solutions at $r>r_{s}$ show that these spectra are almost independent of energy, as expected if the stochastic term in (1) is the leading one. The SWTS (90 AU) spectrum at $T>10$ $\mathrm{MeV} / \mathrm{n}$ now consists mainly of particles that were accelerated far out, at $r \approx 150-170$ $\mathrm{AU}$, due to the $r / r_{b}$ multipliers, and then modulated on their way in. This modulation causes an inflection in the $90 \mathrm{AU}$ spectrum that disappears if the $r / r_{b}$ multipliers in $D$ are taken out. We have verified that this inflection also deepens if the injection energy is lowered or if the compression ratio is increased. We emphasize that the solution of (1) is fully self-consistent, i.e. the only free parameter is the absolute source strength at $0.05 \mathrm{MeV} / \mathrm{n}$, and the spectral forms, specifically the cutoffs, are determined naturally by the various scalings, and are not added artificially or post-hoc. We have verified that latitudinal and drift effects do not alter the solution qualitatively. We note that there is 
a strong positive radial gradient immediately outside the shock, in agreement with the Voyager 1 observations - at $30 \mathrm{MeV} / \mathrm{n}$ the intensity increases with a factor of 5 from 90 to $110 \mathrm{AU}$, for instance. A large part of this observed increase may, however, also be due to demodulation towards solar minimum, as argued for instance by [10] in this issue.

The model does not yet fit the observations quantitatively. The main reason is that this stochastic acceleration process opens up an entirely new parameter regime that we do not yet understand, and that has to be investigated in detail.

\section{SUMMARY}

The re-assessment of energy changes in a heliosphere that contains a termination shock and heliosheath leads us to conclude that: (1) Due to SWTS acceleration and little adiabatic losses in the heliosheath, energy changes are considerably smaller and more dispersive than in a heliosphere without these features. (2) The K-capture electron problem is an example that demonstrates that SWTS acceleration may provide a sensitive tool to probe the shape of low energy $(<100 \mathrm{MeV} / \mathrm{n})$ local interstellar spectra from observations at Earth. (3) Stochastic acceleration in the outer regions of the heliosheath seems like a viable process which, amongst several others, may make a contribution to more complicated spectral shapes in the outer heliosphere than previously anticipated.

\section{ACKNOWLEDGMENTS}

This work was supported by UNAM-DGAPA grant IN106105 in Mexico, NSF grant ATM 0107181 in Maryland, NASA grant NAG5-12929 at Caltech and JPL, and the South African National Research Foundation.

\section{REFERENCES}

1. L. F. Burlaga, Ness, N.F., Acuna, M.H., Lepping, R.P., et al., Science, 309, 2027 (2005)

2. R. A. Caballero-Lopez \& Moraal, H. J.G.R., 109, A01101, doi:10.1029/2003JA10098 (2004)

3. R. A. Caballero-Lopez, Moraal, H., \& McDonald, F.B., Accepted by Geophys. Res. Lett., (2006)

4. R. B. Decker, Krimigis, S.M., Roelof, E.C., Hill, et al., Science, 309, 2020 (2005)

5. L. J. Gleeson \&and I. A. Urch, Astrophys. Space Sci., 25, 387-404 (1973)

6. S. M. Krimigis, Decker, R.B., Hill, M.E., Armstrong, T.P., Gloeckler, G., et al., Nature, 425, 46 (2003)

7. R. A. Mewaldt, Wiedenbeck, M.E., Scott, L.M., et al., AIP Conf. Proc., 719, 127-132 (2004)

8. F. B. McDonald, Stone, E.C., Cummings, A.C., Heikkila, B., et al., Nature, 426, 48 (2003)

9. F. B. McDonald, Cummings, A.C., Stone, E.C., et al., AIP Conf. Proc., 719, 139 (2004)

10. F. B. McDonald, Webber, W.R., Stone, E.C., et al., AIP Conf. Proc., this issue, (2006)

11. S. M. Niebur, Scott, et al., J. Geophys. Res., 108 (A10), 8033, doi:10.1029/2003JA009876(2003)

12. V. Ptuskin, Moskalenko, I.V., Jones, F.C., Strong, et al. accepted Astrophys. J. (2006)

13. C. D. Steenberg, \& Moraal, H., J. Geophys. Res., 104, 24879 (1999)

14. E. C. Stone, Cummings, A.C., McDonald, F.B., Heikkila, B.C., et al., Science, 309, 2017 (2005)

15. N. E. Yanasak, Wiedenbeck, M.E., Mewaldt, R.A., Davis, et al., Astrophys. J., 563, 768 (2001) 